\title{
Effect of a stimulus paired with reinforcement as a function of reinforcement magnitude
}

\author{
JOHN G. CARLSON \\ University of Hawä̈, Honolulu, Hawä̈ 96822
}

\begin{abstract}
A leverpressing response of rats conditioned with one food pellet in the presence of a light was disrupted when an auditory stimulus was presented that had been previously paired with eight pellets. The results were discussed in terms of a model stressing stimulus properties of anticipatory states.
\end{abstract}

Traditionally, the effects of magnitude of reinforcement have been explained in motivational terms. According to Spence (1956), for example, a stimulus antecedent to a goal becomes conditioned to evoke $r_{g}$, a fractional component of the primary response to the goal object. In turn, the occurrence of $r_{g}$ is said to contribute to an animal's level of incentive motivation. Spence further suggested that one determinant of the strength of $r_{g}$ is amount of reinforcement. A large amount of food, for example, should condition a stronger $r_{g}$ and a higher level of incentive than a smaller amount of food.

However, a study by Hyde, Trapold, and Gross (1968) failed to confirm one of two predictions from the incentive position. Rats were first trained to press a response lever for food at the onset of a discriminative stimulus. Next, with the response lever removed, for one group (prepaired) one auditory stimulus was paired with 10 pellets of food and a second auditory stimulus was paired with one pellet. For another group the stimuli and differing amounts of food were presented but never explicitly paired. Finally, the response lever was again made available and one or the other auditory stimulus was substituted for the light on occasional trials. The animals in the prepaired group came to press the lever more quickly to the auditory stimuli than the other animals, as would be expected from incentive theory. That is, presumably the pairings between the stimuli and food for the prepaired group conditioned $r_{g} s$ to these stimuli. Therefore, relative to the other group, levels of incentive of the prepaired animals should have been higher in the auditory stimuli. On the other hand, contrary to what would have been expected if different levels of incentive were conditioned to the two stimuli, Hyde et al. failed to find shorter latencies in the stimulus prepaired with the larger amount of food than in the other stimulus.

In their discussion, Hyde et al. suggested that perhaps $r_{\mathbf{g}} s$ were established to the auditory stimuli during the

Reprint requests should be sent to John G. Carlson, Department of Psychology, University of Hawaii, Honolulu, Hawaii 96822 . prepairing phase but that internal stimulus feedback, $\mathrm{s}_{\mathrm{g}} \mathrm{s}$, generated by these responses was more prevalent than potential incentive properties. This explains the enhanced transfer of leverpressing from the discriminative stimulus to the food-paired stimuli in the prepaired group; leverpressing should have been associated with stimulus feedback from the foodrelated $r_{g} s$ during the discriminative training and now elicited by the auditory cues in the test phase. However, it is further suggested that perhaps "the $s_{\mathbf{g}}$ resulting from a 10-pellet $r_{g}$ is not appreciably different from a 1-pellet $r_{g}$ " (Hyde et al., 1968, p. 427). This would explain the failure of the auditory cue previously paired with 10 pellets to enhance performance more than the cue previously paired with one pellet, as predicted by incentive theory.

Another possibility is that some aspect of the Hyde et al. procedure was responsible for their negative results. At least two features of their methods are notable. First, when the investigators tested for transfer of leverpressing to the food-paired stimuli, responding was reinforced with one pellet in the presence of both stimuli. Theoretically, this would have resulted in the conditioning of an anticipation based upon the low-magnitude reinforcer to both stimuli, possibly attenuating any previously established differences between the cues.

A second possible reason for the negative results of the Hyde et al. study relates to the fact that, because the test stimuli were presented in the absence of the discriminative stimulus during generalization testing, response latencies were initially very long on these trials. The authors stated that prior training had established absence of the discriminative cue as a very effective S-delta for lever approach. It is conceivable that initial unresponsiveness of the animals in the test stimuli masked differential effects of these cues established in the prepairing phase.

For these reasons, the present experiment was designed as a replication of the Hyde et al. experiment with the following major changes. One, responding on test trials was not reinforced at all to prevent possible rapid conditioning of new anticipations to the test stimuli. Two, the test stimuli were superimposed 
upon a previously established discriminative stimulus to assure that there would be some pre-established tendency of the animals to press the lever on these trials.

\section{METHOD}

\section{Subjects}

Sixteen male albino rats, approximately 100 days old at the start of the study, were maintained at $80 \%$ of freefeeding body weight.

\begin{abstract}
Apparatus
Two standard conditioning chambers were housed in sound- and light-attenuating cubicles. Each chamber was equipped with a retractable response lever on one wall, a houselight located in the back of the cubicle, a small loudspeaker, and a pellet feeder that dispensed $45-\mathrm{mg}$ food pellets (Noyes, Formula A) into a trough located approximately $85 \mathrm{~mm}$ to the right of the lever. Ambient white noise in the room housing the chambers masked the sound of control and recording equipment in another room.
\end{abstract}

\section{Procedure}

The subjects were randomly assigned to the chambers, adapted, magazine and leverpress trained. Next, the lever was retracted from the chamber and half of the subjects in each chamber were randomly assigned to one of two groups. For the paired group $(n=8)$, a tone (approximately $1000 \mathrm{~Hz}$ ) and click (approximately six clicks/sec) were each sounded 12 times in each of 24 sessions for $3 \mathrm{sec}$, at varying intervals of time averaging $1 \mathrm{~min}$. For half the subjects in this group, presentations of the click (the high-magnitude stimulus) terminated in the delivery of eight food pellets, and presentations of the tone (the low-magnitude stimulus) were followed by the delivery of one pellet. For the remaining subjects in this group, the tone was paired with eight pellets, the click with one pellet. For the unpaired group $(n=8)$, the tone and click were presented on the same schedule of varying intervals employed for the paired group; however, deliveries of reinforcement (12 of one pellet, 12 of eight pellets per session) were on a separate and independent schedule of varying intervals averaging $1 \mathrm{~min}$. (This procedure provided the random control for classical conditioning suggested by Rescorla, 1967.)

Next, the response lever was again made available for six sessions, and the subjects were trained on the following discrimination problem. At varying intervals averaging $1 \mathrm{~min}$, the houselight was turned on. The first press of the lever in the presence of the light delivered a single pellet. The houselight was turned off within approximately $1 \mathrm{sec}$ of reinforcement and the timing of the next intertrial interval was begun. In addition, any leverpress within $15 \mathrm{sec}$ prior to the onset of the houselight postponed the beginning of the next trial for an additional $15 \mathrm{sec}$, a procedure that rapidly eliminated between-trials leverpressing. A trial was automatically terminated $60 \mathrm{sec}$ from onset if no leverpress was made. The time elapsed from the beginning of a trial (designated by onset of the houselight) to a leverpress defined the latency of responding. Latencies were cumulated across 25 trials per session and the average computed for each animal.

Finally, during transfer training, in addition to the light, either the tone or click was presented on Trials 7, 13, 19, and 25. The two stimuli were alternated from one presentation to the next, for instance, tone, click, tone, click. For half of the subjects in each group, the first stimulus presented on the first day was the tone, on the following day, the click. For the remaining subjects, the order of stimulus presentations beginning on the first day was reversed. The test of the auditory stimuli was continued for six sessions until mean latencies in the light plus high-magnitude stimulus nearly equaled those in the light plus low-magnitude stimulus for the paired group.

\section{RESULTS AND DISCUSSION}

Figure 1 depicts the latencies for both groups in discrimination training and transfer testing. A logarithmic transformation of the data was made in order for the distributions to more closely meet the assumptions of the statistical tests. The figure shows means across all daily trials on which the light alone was presented. During testing for the paired group, the figure also shows latencies on each of the trials on which the light was combined with either the highmagnitude or low-magnitude stimulus. During testing for the unpaired group, means are shown in order from left to right for the first two and last two daily trials on which the light was combined with the auditory stimuli.

During discrimination training the two groups rapidly attained relatively short and stable response latencies in the presence of the light alone. These levels were maintained throughout the subsequent transfer test. By contrast, in the transfer phase presentations of the high-magnitude stimulus to the paired group caused a marked increase in latencies, an effect that tended to diminish across test sessions. There were no consistent differences among the latencies to the light plus low. magnitude stimulus for the paired group, latencies to the auditory stimuli for the unpaired group, and the latencies on light-only trials for either group. Since no reinforcements were delivered on test trials, the similarity between latencies on these trials and latencies on light-only trials apparently reflects a strong generalization effect.

With the significance level set at .05 , an analysis of variance was performed on test trials only, with groups, reinforcement magnitude, trial sequence (that is, first and second presentations of a stimulus in each session), and sessions as factors. (For the unpaired group trials with the auditory stimuli that were comparable in sequence to the high- and low-magnitude stimulus trials for the paired group served for the comparison between groups.) The differences between the treatments apparent in the figure were confirmed in a significant groups effect, $F(1,14)=10.60, p<.007$ reinforcement magnitude effect, $F(1,14)=20.62$, $\mathrm{p}<.0005$, and Groups by Magnitude interaction, $F(1,14)=18.71, p<.0008$. All other main effects and interactions were nonsignificant.

In follow-up contrasts there was no significant difference between the mean of trials on which the low-magnitude stimulus was presented to the paired group and the mean for the trials on which the auditory stimuli were presented to the unpaired group $(F<1.00)$. 


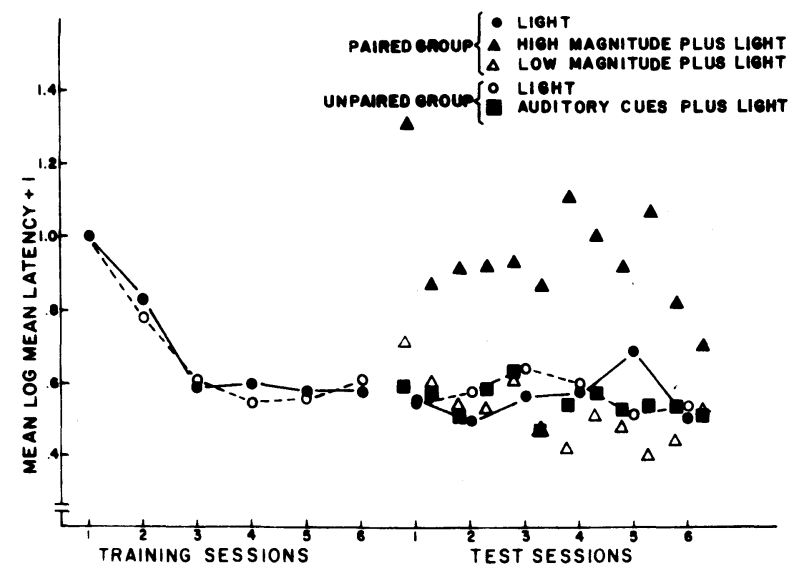

Figure 1. Means of logarithmic transformations of mean daily latencies for each group in discrimination training (left half of figure) and transfer testing (right half of figure). See text for explanation.

However, the mean for trials on which the highmagnitude stimulus was presented to the paired group differed significantly from the mean for trials on which the low-magnitude stimulus was presented and from the mean for auditory stimulus trials for the unpaired group $(\mathrm{p}<.0005)$.

Therefore, these methods proved somewhat more sensitive than those of Hyde et al. (1968) in showing differential effects upon instrumental responding of stimuli prepaired with different amounts of positive reinforcement. Moreover, the results obtained with the paired group were completely inconsistent with a prediction from incentive theory of magnitude of reinforcement; rather than enhancing performance, the stimulus paired with the large amount of food produced a decrement in performance relative to the stimulus paired with the small amount of food.

These results are consistent with an interpretation of the effects of magnitude of reinforcement different from both the incentive position and that offered by Hyde et al. (1968). In terms of a recent "central" mediational account (Trapold \& Overmier, 1972), it may be argued that different amounts of reinforcement condition distinctively different "expectancies" to reinforcement-paired stimuli. From this view it follows that, in the discrimination training of the first phase of this experiment, leverpressing came under the control of "stimulus feedback" arising from an expectation of one pellet in addition to the external discriminative stimulus. In the next phase, distinctively different expectations of one pellet and eight pellets were established to the respective auditory stimuli in the paired group. In the final test phase, presentations of the one-pellet paired stimulus would have evoked the same expectancy as that already conditioned to the light and associated with leverpressing. Therefore, there would have been a substantial tendency for leverpressing to transfer to the combination of this auditory cue and the light. By contrast, presentations of the eight-pellet paired stimulus would have evoked a distinctively different expectancy and one not previously associated with leverpressing. As a result, responding to the combination of this cue and the light would have been disrupted through the introduction of new or altered stimuli to the complex of cues available to an animal. This interpretation has additional support in other recent studies of the effects of stimuli correlated with different magnitudes of reinforcement (Carlson \& Wielkiewicz, in press; Gross, 1971).

However, other interpretations of these data are possible. A traditional mediational approach would "locate" the mediators more peripherally than the central or cognitive mechanisms of recent theories. By emphasizing $\mathbf{s}_{\mathbf{g}}$ characteristics rather than incentive properties of the traditional $r_{g}$, the present data could be handled in terms otherwise similar to the "expectancy" interpretation outlined above. By contrast with these approaches, it could also be argued either that operants involved in food-getting or perhaps a more general "emotional state" (Azrin \& Hake, 1969) became conditioned to the auditory cues in the pairing phase and later interfered with leverpressing in the test phase. In order for the latter models to account for the differential effects of the two test stimuli, it would also have to be assumed that the strength of such incompatible responses, operant or respondent, was a function of the amount of reinforcement used in the pairing phase. A systematic evaluation of these alternative approaches appears to be in order in view of the significance of the present results for traditional concepts of magnitude of reinforcement as well as for a more general understanding of mediational mechanisms in animals.

\section{REFERENCES}

Azrin, N. H., \& Hake, D. F. Positive conditioned suppression: Conditioned suppression using positive reinforcers as the unconditioned stimuli. Journal of the Experimental Analysis of Behavior, 1969. 12, 167-173.

Carlson, J. G., \& Wielkiewicz, R. M. Mediators of the effects of magnitude of reinforcement. Learning and Motivation, in press.

GRoss, D. M. Learned expectancies in rats based on differential magnitudes of reinforcement. (Doctoral dissertation, University of Minnesota.) Ann Arbor, Mich: University Microfilms, 1971. No. 71-28239.

Hyde, T. S., Trapold, M. A., \& Gross, D. M. Facilitative effect of a CS for reinforcement upon instrumental responding as a function of reinforcement magnitude: A test of incentivemotivation theory. Journal of Experimental Psychology, 1968, 78, 423-428.

Rescorla, R. A. Pavlovian conditioning and its proper control procedures. Psychological Review, 1967, 74, 71-80.

SPENCE, K. W. Behavior theory and conditioning. New Haven: Yale University Press, 1956.

Trapold, M. A., \& Overmier, J. B. The second learning process in instrumental learning. In A. H. Black and W. F. Prokasy (Eds.), Classical conditioning II. New York: Appleton-Century-Crofts. 1972. Pp. 429-452.

(Received for publication November 10. 1975.) 